Available online on 15.02.2019 at http://jddtonline.info
Journal of Drug Delivery and Therapeutics
Open Access to Pharmaceutical and Medical Research
$\begin{gathered}\text { (c)11-18, publisher and licensee JDDT, This is an Open Access article which permits unrestricted } \\ \text { non-commercial use, provided the original work is properly cited }\end{gathered}$

Open $\mathcal{O}_{\text {Access }}$

Case Study

\title{
Infection of Mycobacterium mageritense at surgical site: A first case report of India
}

\author{
*1Bhavini S Shah, ${ }^{6}$ Harsh Toshniwal, ${ }^{1}$ Hari Shankar P Ray, 1,2Parth S Shah, 1,3Nidhi D Shah, 1Nikunj B Khatri, ${ }^{6}$ Harsh Shah, \\ ${ }^{5}$ Vipul V Shah , ${ }^{7}$ Baldev V patel , 1 Sandip C Shah and ${ }^{1,4}$ Mandava V Rao \\ ${ }^{1}$ Department of Microbiology Supratech Micropath Laboratory and Research, Institute, Ahmedabad, India \\ ${ }^{1,4}$ Ex-Director, School of Sciences, Gujarat University, Ahmedabad, Gujarat-India \\ 1,2 Department of Medicine, Lahey Hospital and Medical Center, Boston, Massachusetts USA \\ 1,3 Department of Pediatrics, Nassau University Medical Centre, New York City, New York, USA. ${ }^{5}$ Healthcare Infectious Disease Clinic, Ahmedabad, \\ India \\ ${ }^{6}$ Infectious Disease and Tropical Clinic (IDTM-clinic), Ahmedabad India \\ ${ }^{7}$ Professor, Department of Microbiology and Biotechnology, School of Sciences Gujarat University, Ahmedabad, Gujarat, India
}

\begin{abstract}
A 27 years old non diabetic, normotensive, female had undergone lower segment caesarean section (LSCS) with tubular ligation. She complained discharge at left side of stitch line after about 5 weeks of operation, and was treated with broad spectrum antibiotics. As she did not improve, first and second debridement was done in January and February 2017 and pyogenic cultures sent were sterile. Later she was advised for Multiple detector computed tomography (MDCT) in March 2017, which revealed accumulations in pre-peritoneal space of urinary bladder posteriorly and reached to anterior wall of uterus. This pus was from multiple sinuses and were sent for mycobacterial cultures which showed growth of MOTT- M. mageritense further identified by MALDI-TOF and supported by molecular technologies. After identification of this Nontuberculosis mycobacteria (NTM). She was treated for the same and recovered completely after seven months of anti-NTM treatment. This is the first reported case of M. mageritense in skin and soft tissue in India. Literature also reports a few cases around the globe.
\end{abstract}

Keywords: Mycobacterium mageritanse, Tubular ligation Lower segment Cesarian section (LSCS), Microbial culture, Rapid molecular techniques, MDCT, antibiotic treatment.

Article Info: Received 14 Jan 2019; $\quad$ Review Completed 10 Feb 2019; $\quad$ Accepted 10 Feb 2019; Available online 15 Feb 2019

\section{Cite this article as:}

Shah BS, Toshniwal H, Ray HSP, Shah PS, Shah ND, Khatri NB, Shah H, Shah VV, Patel BV, Shah SC, Rao MV, Infection of Mycobacterium mageritense at surgical site: A first case report of India, Journal of Drug Delivery and Therapeutics. 2019; 9(1-s):369-373 DOI: http://dx.doi.org/10.22270/jddt.v9i1-s.2386

*Address for Correspondence:

Bhavini S Shah, Department of Microbiology Supratech Micropath Laboratory and Research, Institute, Ahmedabad, India

\section{INTRODUCTION}

Mycobacterium mageritense, belongs to non tuberculosis mycobacterial group. It is similar to rapidly growing pigmentation types, which has a close resemblance to $\mathrm{M}$. fortuitum complex ${ }^{1,2}$. It causes human disease like furunuculosis, which was reported by Gira et al 3 and Winthrop et al ${ }^{4}$ detected this bacteria in cases working in nail salon where they received foot-baths prior to pedicures. The above microbe was isolated by pulsefield gel electrophoresis including environmental isolates and water 5,6 . Two isolates of sputum in Spain, six strains from USA, isolated from sputum, bronchial fluid, blood, sinus drainage and surgical wound infection were noticed. These were also similar to that of M. fortuitum third biovariant complex ${ }^{7-9}$. Isolation of these bacteria from lung of a harbor porpoise

ISSN: 2250-1177 with severe granulomatous lesions was causative agent for its death ${ }^{10}$. There have been few reports in relation to skin and soft tissue infections due to these bacteria outside India $3,6,7,11,12$.

Studies on Post-surgical inoculation wound infection / Post infection abscess in India have isolated NTM from various sites like skin and soft tissue after laparoscopic procedure. Typical manifestation is post laparoscopic non healing wound since months or years, resulting in chronic discharge sinus tracts. The discharge is usually serous in nature. The lesions are usually painless. Fever is usually absent. Empirical antibiotics and antituberculous agents are tried without much benefit in such cases. Hence, this study was undertaken to report the first case with M. mageritense from 
soft tissue at surgical site during tubular ligation of a case from India.

\section{CASE REPORT}

A 27 old woman underwent LSCS with tubular ligation on $9^{\text {th }}$ October 2016. About 5 weeks, she complained of discharge from left side of stitch line. She was then treated with antibiotics and daily dressing. Antibiotics were Tab. Cephalaxin for 15 days, Tab. Cefexime for 10 days and Tab. Amoxicillin with clavulinic acid for 7 days. Since, she did not improve; first debridement was done in January.2017, followed by second debridement in February 2017.Pus sent for pyogenic culture gave negative report. The Gram stain and ZN stain were reported negative. Both debridements were done at sub district place. Then MDCT was advised. It revealed collection accumulated in a well near infraumbical region, reaching urinary bladder posteriorly and uterine wall interiorly. Other organs like liver, spleen, kidney and urinary bladder appeared normal. However, the thick walled loculated (pus) collections in anterior abdominal wall and subcutaneous plane in infraumbilical region reaching into extra peritoneal space of pelvic cavity up to anterior wall of uterus were used for cultures and rapid molecular techniques to detect specific mycobacterium causing infection at surgical site. Latest diagnostic technologies were used for precise identification of the isolate at Supratech Micropath Laboratory and Research Institute, Ahmedabad ${ }^{13}$. Following fig.1

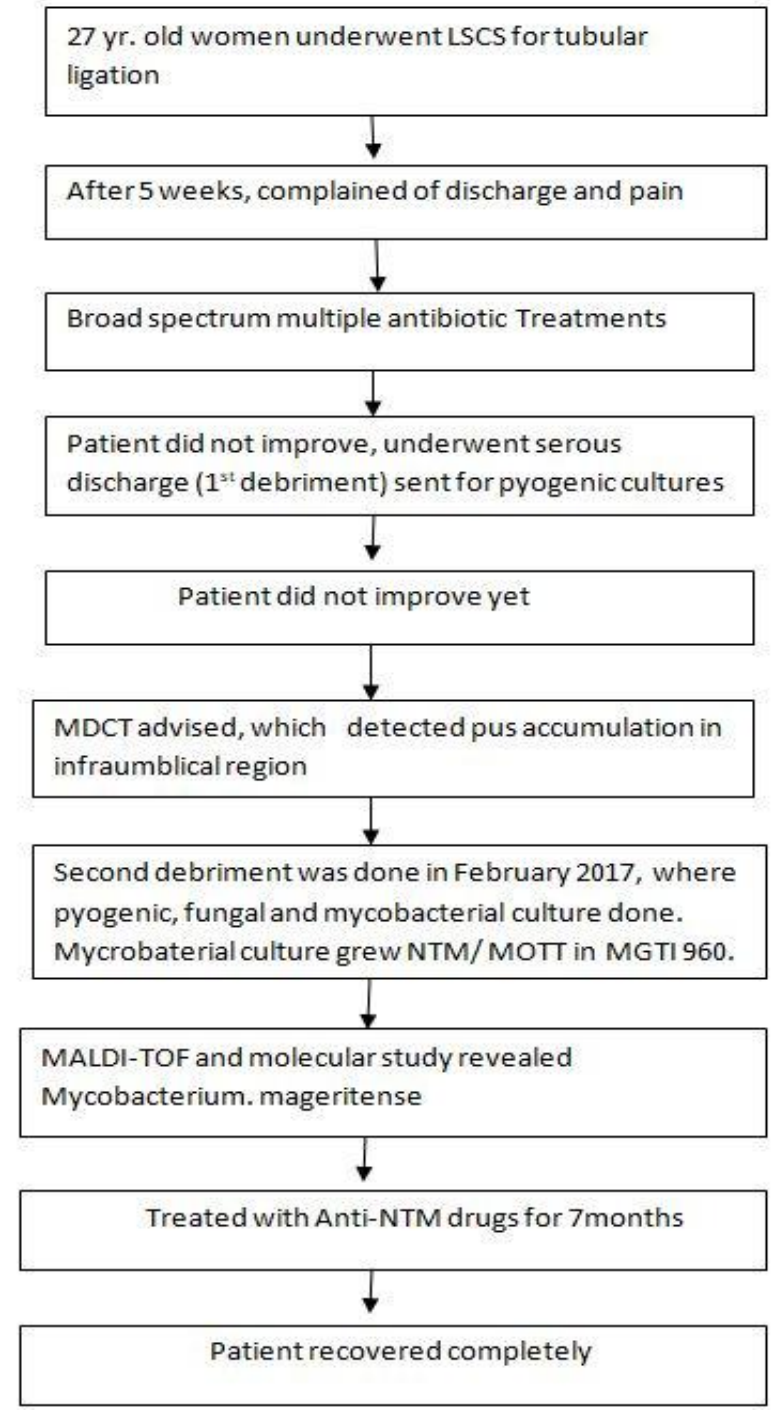

Figure 1: Flow chart of series of events from surgery to recovery

ISSN: 2250-1177

\section{MATERIALS AND METHODS}

\section{Microbiological Examination}

Potassium hydroxide $(\mathrm{KOH})(10 \%)$ examination was done on tissue slide and was examined to identify fungal elements. Gram stain procedure was adopted for detecting gram positive and gram negative bacteria in our sample following standard method in our laboratory. Ziehl-Neelsen stain (ZNCF) stain was also performed on the specimen to detect presence of AFB. Further pyogenic culture was performed by standard method following the protocol in our laboratory, whereas the fungus culture was included and incubated at two different temperatures $25^{\circ} \mathrm{C}$ and $35^{\circ} \mathrm{C}$ to isolate majority fungal types. AFB Culture was performed by liquid culture medium by MGIT 960 .

\section{LPANTM CM (Line Probe Assays Nontuberculous Mycobacteria Common Mycobacteria)}

The genotype Mycobacterium CM test is based on the DNASTRIP technology. The whole procedure is divided into three steps involving DNA extraction from cultured material liquid medium, a multiplex amplification with biotinylated primers and a reverse hybridization. All reagents needed for amplification, such as polymerase and primers, are included in the Amplification Mixes A and B (AM-A and AM-B) and are optimized for this test. The membrane strips are coated with specific probes complementary to the amplified nucleic acids. After chemical denaturation, the single-stranded amplicons bind to the probes (hybridization). Highly specific binding of complementary DNA strands are ensured by stringent conditions which result from the combination of buffer composition and certain temperature. Thus the probes reliably discriminate the different sequences of the bacterial species. The streptavidin-conjugated alkaline phosphatase binds to the amplicons' biotin via the streptavidin moiety. Finally, the alkaline phosphatase transforms an added substrate into a dye which becomes visible on the membrane strips as a colored precipitate. A template ensures the easy and fast interpretation of the banding pattern obtained.

\section{MALDI-TOF (Matrix Assisted Laser Desorption Ionization - Time of Flight)}

It is based on three functional units having ion source, mass analyzer and detection device to monitor sample ions which follow a simple protocol. The pure growth from positive Mycobacteria Growth Indicator Tube (MGIT) culture tube was taken as sample identification on MALDI-TOF (Bruker's microflex) to score for detection of the bacteria.

\section{Molecular analysis}

\section{DNA extraction}

The positive MGIT culture isolate was used as a specimen for molecular analysis. Sonicated Mycobacterial cells were use for bacterial DNA extraction using manually DNA extraction Kit (Roche high pure kit, India). The concentration of DNA was determined using the Qubit 4.0 fluorometer HS DNA kit (Thermo Fisher Scientific, USA).

\section{Sanger DNA sequencing}

The 16S rRNA gene was designed to determine properties of designed primers ${ }^{14}$ and also we are using Primer Blast (https://www.ncbi.nlm.nih.gov/tools/primer-blast/) to check whether our region of interest is covered or not. Primers were synthesized at $100 \mathrm{pM}$ (Picomole) scale and cartridge purified (Eurofins India). Polymerase Chain Reaction (PCR) amplification was carried out using rTaq Premix Taq Version 2.0 PCR master mix with 8F and 1492R, CODEN (USA): JDDTAO 
$16 \mathrm{~S}$ rRNA gene specific primers at $10 \mathrm{pM}$ concentration under standard conditions. PCR amplifications were all carried out in duplicate alongside a known normal control cell line sample. Successful PCRs were purified prior to sequencing using ExoSAP-IT reagent (Affrymetrix-USA) according to the manufacturer's protocol. Purified PCR products were Sanger sequenced in both forward and reverse orientations using the same primer sequences used for PCR at $10 \mathrm{pM}$ final concentration using BigDye ${ }^{\circledR}$ v3.1 according to manufacturer's cycling conditions. BigDye ${ }^{\circledR}$ v3.1 sequencing reactions were then purified using Ethylene diamine tetra acetic Acid (EDTA) and sodium acetate according to the manufacturer's protocol and analyzed on 3500 Genetic Analyzer (Applied Biosystems USA). Sanger sequencing data was analyzed using Codon Code Aligner v5.0.2, (Codon Code Corporation Centerville, USA) and NCBI blast the entire sequence.

\section{Next generation sequencing (NGS)}

For targeted NGS analysis, 16S rRNA gene primers were used. PCR was performed using 5-15 ng/ $\mu \mathrm{l}$ bacterial DNA and rTaq Premix Taq Version 2.0 PCR master mix (TAKARA) for initial denaturation at $95^{\circ} \mathrm{C}$ for $10 \mathrm{mins}$, followed by 35 cycles of denatuation at $95^{\circ} \mathrm{C}$ for $30 \mathrm{sec}$, annealing at $52^{\circ} \mathrm{C}$ for $4 \mathrm{~min}$ and elongation at $72^{\circ} \mathrm{C}$ for 2 mins. Final elongation at $72^{\circ} \mathrm{C}$ for 10 mins. and holding period at $4^{\circ} \mathrm{C}$. About1.4 kb PCR amplicons was purified using (1.4x) Agencourt AMPure XP reagents (Beckman Coulter, USA) to remove the primer dimer. For End repair and fragmentation use 100ng/ $\mu \mathrm{l}$ of DNA concentration and incubate the end-repair reaction for 20 minutes at room temperature using the Ion plus fragment kit (Life technology-USA). Amplicons purified using Agencourt AMPure XP reagents. The amplicons were ligated to adapters with barcodes of the Ion XpressTM Barcode Adapters kit (Life Technologies, USA) for 15 mins at $25^{\circ} \mathrm{C}$ then $72^{\circ} \mathrm{C}$ for 5 min and hold at $4^{\circ} \mathrm{C}$. Adapter ligated amplicon libraries were purified using Agencourt AMPure XP reagents (Beckman Coulter, USA). The library concentration was determined using an Ion Library Quantitation Kit (Life Technologies, USA), then each library was diluted to $4-8 \mathrm{pmol} / \mathrm{L}$ and the same amount of libraries was pooled for one sequence reaction. Next, emulsion PCR was carried out using the Ion OneTouchTM 2.0 System and Ion 540TM OT2 Reagents (Life Technologies, USA) according to the manufacturer's instructions. Template-positive Ion SphereTM Particles were then enriched with Dynabeads MyOneTM Streptavidin C1 Beads (Life Technologies, USA) using an Ion OneTouchTM ES system (Life Technologies, USA). Purified Ion Sphere particles were loaded on 540 (Compatible with Ion S5 System) Chip. Massively parallel sequencing was carried out on Ion S5 sequencer (Thermo Fisher, USA) using Ion S5 Sequencing kit according to the manufacturer's instructions. Sequencing was performed using 500 flow runs that generated approximately $200 \mathrm{bp}$ reads.

\section{Data analysis}

The data generated from the Instruments are transferred to the Standalone Torrent Suite (version 5.2) analysis server as Direct agglutination test (DAT) files. This DAT files contains electrical signals generated during sequencing flow. The Torrent Suite software (version 5.2) coverts this electrical signals into numerical one and store them in 1.wells files during the signal processing step. BaseCaller will read the information stored in 1.wells file and covert them into sequence of bases and computes the quality scores of each base. The next step is barcode filtration and adapter, low quality reads, low signal bases trimming. The final data is stored in an unaligned Binary Alignment Map (BAM) file which can be used for further alignment processes. The unaligned BAM files are downloaded from the Torrent Suite software (version 5.2) and converted into FASTQ format file to run with BWA aligner (version 0.7.12-r1039). BurrowsWheeler Aligne (BWA) aligner is used to align the raw reads to human reference genome hg19 to remove human reads from the data. The unaligned non-human reads are then extracted from the BWA output file and stored in BAM format file. Newly generated BAM files are then uploaded to the standalone Ion Reporter software (version 5.2) for identifying the presence of bacterial genome present in the data. The uploaded BAM files are run through the Metagenomics 16S w1.1workflow of Ion Reporter (version 5.2) for the identification of species present in the data. Simultaneously, the reads in a BAM files are extracted with custom script and stored in a Federal Acquisition Streamlining Act (FASTA) file for running with standalone Basic Local Alignment Search Tool (BLAST) program. The standalone blast is run against "nr" database to reconfirm the results obtained by Ion Reporter software (version 5.2).

\section{RESULTS AND DISCUSSION}

M. mageritense was first isolated by Domenech et $\mathrm{al}^{1}$, Similar to others like M. fortuitum and M. abscessus. This organism is linked with human diseases as reported in USA ${ }^{3}$. It is rapidly growing bacteria like others and is ubiquitous and also usually isolated from Municipal water 2. Few reports have also been described of skin and soft tissue infections due to this mycobacterium outside India. In our patient, this isolate was identified in a patient who underwent LSCS with tubular ligation in October 2016. There was disgorge from left side of stitch line after 5 weeks of postop period. Treatment of broad spectrum antibiotics was given, but collection of discharge (pus) persisted even after two subsequent debridements in January and February 2017. Then MDCT report detected pus in between abdominal anterior wall and infraumbilical region reaching to anterior wall of uterus. Thus, this isolate was examined using microbiological culture tests and molecular techniques. The clinical specimen underwent $\mathrm{KOH}$ examination; Gram stain and ZNCF stain which were negative. Pyogenic and fungus culture were also negative. The clinical specimen was run on Mycobacteria Growth Indicator Tube (MGIT 960) system, an automated liquid media system for AFB culture, which was positive for Nontuberculous Mycobacteria other than tuberculosis (NTM/MOTT). Gene expert was also negative. The pure growth of NTM/MOTT was subjected to LPANTM $\mathrm{CM}$, a Line probe assay for identification of common NTM/MOTT. The results showed good control bands but did not show any relevant conclusive identification bands. Further the sample was subjected to MALDI TOF where the sample was identified with score of 2.27 as M. mageritense (Fig.2). Further the other molecular techniques were adopted for genome analysis of these bacteria. The analysis of 16s rRNA using Sanger sequencing and Next generation Sequencing (NGS) revealed that the two pictures observed in both techniques (Figs 3a,3b) were found to be similar to that of NCBI strains CS33.1(Fig.4). Similarly the draft genome sequence of M. mageritense species/strains DSM $44476^{\mathrm{T}}$ of NTM causing various infections was available ${ }^{5}$. Refined analysis incorporating the sequence of 16r RNA and other genes indicated that $M$. mageritense stands by itself as a separate species. Our results on 16s rRNA extracted from our isolate also indicated the same microbe as cited above. All these results indicated that this strain is a novel one separate from other types and is the first isolate report in India. This investigation thus was further supportive to the study of MALDI-TOF. In clinical sites or samples which have not responded after multiple uses of broad spectrum antibiotics, surgical debriment and these are no positive 
finding in pyogenic microbiological cultures, a ZN stain and AFB culture should be considered. Newer technologies play an important role in identification of novel bugs. In this MALDI-TOF helped the case in identification the mycobacterial species along with molecular technique and planning the NTM therapy. Such technologies will also be of great help to develop epidemiology of local regions.

\begin{tabular}{ll} 
& $\begin{array}{c}\text { IDENTIFICATION BY MALDI TOF } \\
\text { (Mass Spectrometry) }\end{array}$ \\
\hline $\begin{array}{l}\text { Specimen } \\
\text { * IDENTIFICATION BY MALDI TOF } \\
\text { (MasS Spectrometry) }\end{array}$ & $\begin{array}{l}\text { PUS SPECIMEN } \\
\text { Mycobacterium mageritense (Identified on MALDI TOF , score : 2.27) }\end{array}$ \\
\hline
\end{tabular}

Figure 2: Report of M. mageritense score from MALDI-TOF

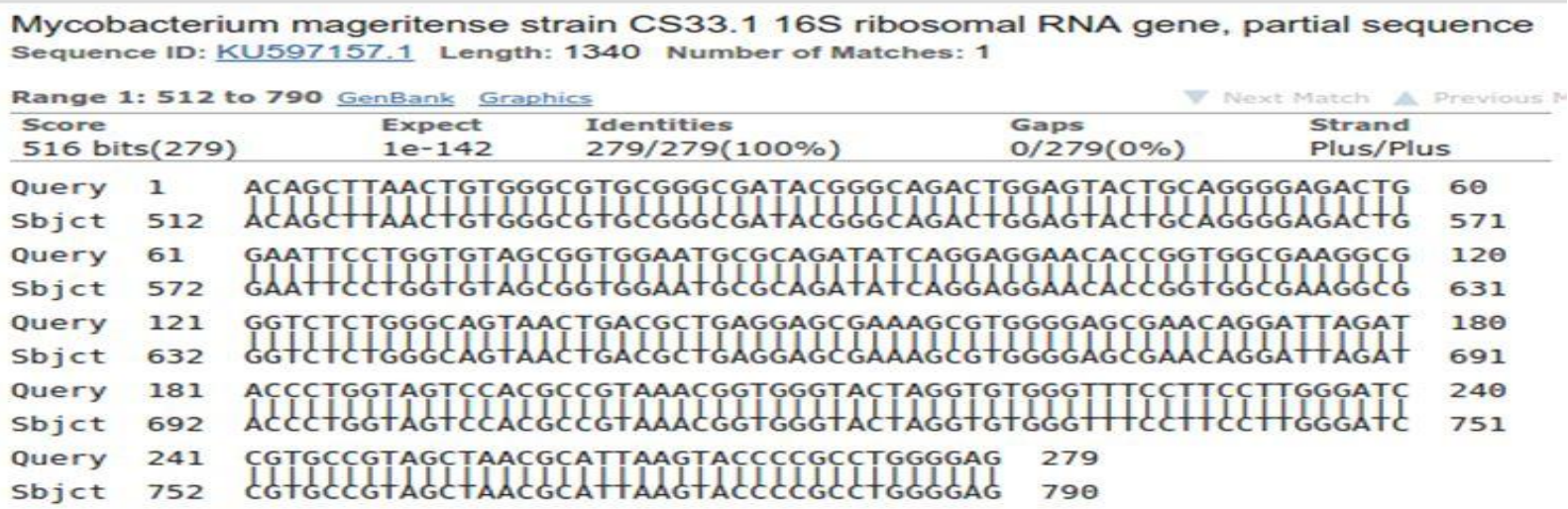

Figure 3a: NCBI Blast from NGS showing sequence of sequence 16S rRNA gene (strain CS33.1).

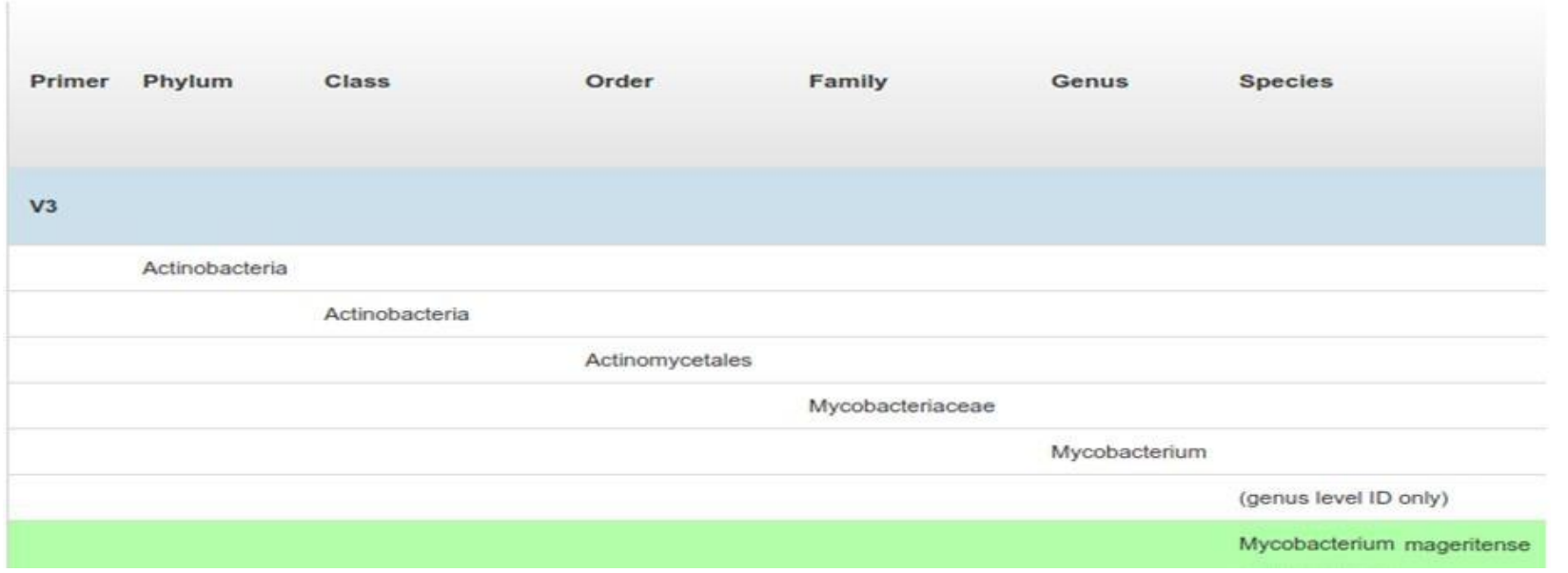

Figure 3b: IR suite showing species level identification of bacteria

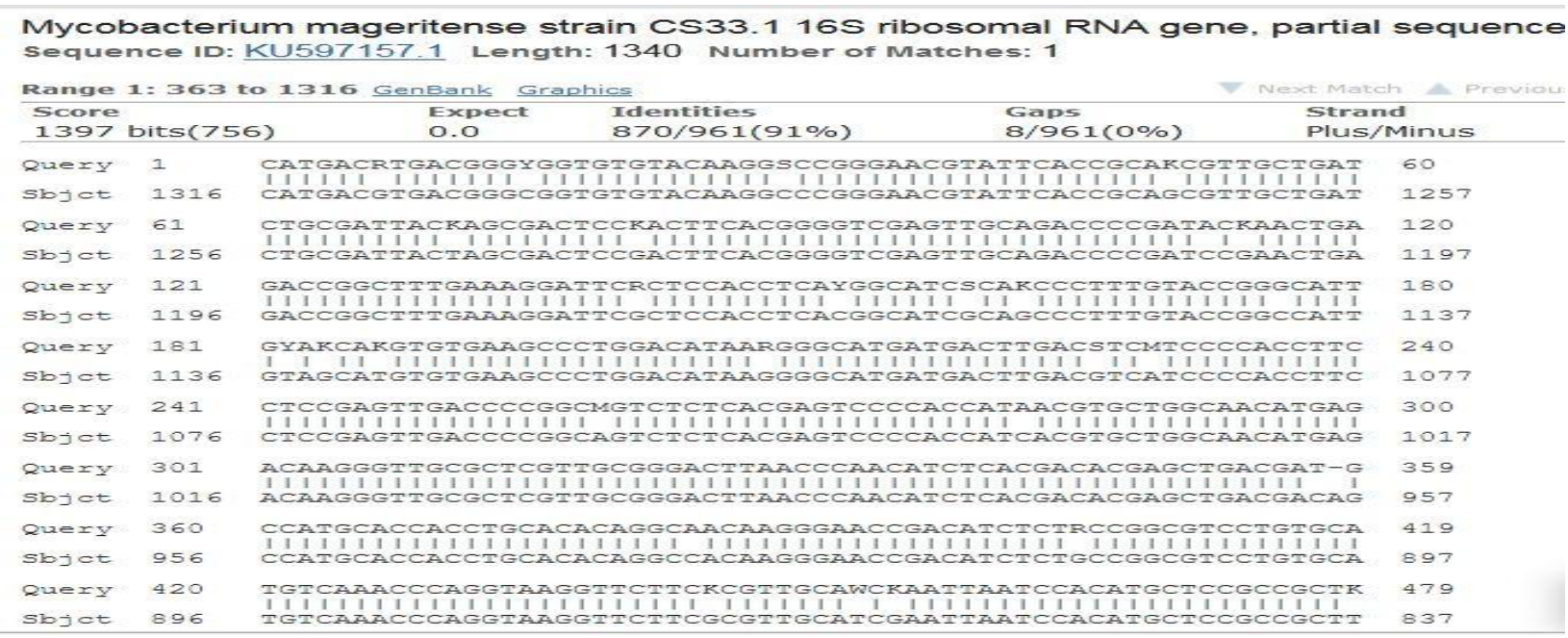

Figure 4: NCBI blast result of Sanger sequencing of strain CS33.1 16s rRNA gene. 


\section{TREATMENT}

After confirmation of the strain, Cephalaxin, Cefixime and Amoxicillin with clavulinic acid viz i.e anti NTM treatment for 7 months was suggested for complete recovery.

\section{CONCLUSION}

Non- NTM strain, M. mageritense was detected in pus collected from a well formed from soft tissue as a result of

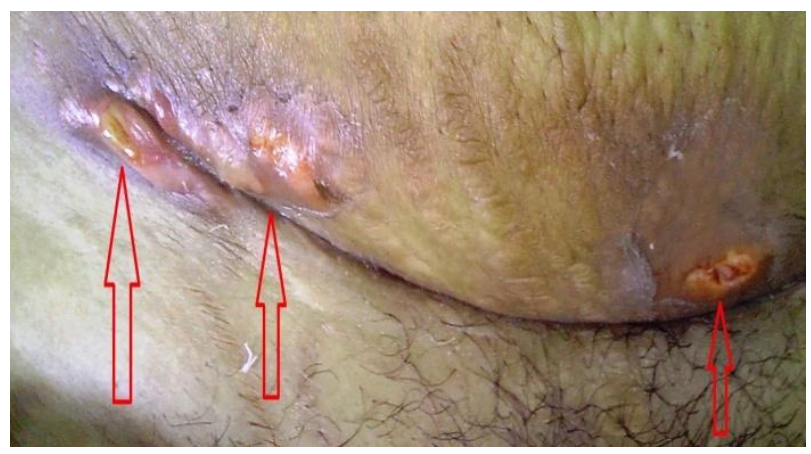

Figure 5a: Before treatment

\section{CONFLICT OF INTEREST}

There are no conflicts of interest among authors

\section{ACKNOWLEDGMENT}

We sincerely thank all staff of Supratech Laboratory and IDTM clinics for their assistance and cooperation in this study.

\section{REFERENCES}

1. Domenech P, Jimenez MS, Menendez MC, Bull TJ, Samper S, Manrique A, Garcia MJ, Mycobacterium mageritense sp. nov, Int J Syst Bacteriol, 1997; 47(2):535-540.

2. Ogawa Y, Soichiro I, Nakano R, Kasahara K, Kuwahara M, Yano $\mathrm{H}$, Mikasa $\mathrm{K}$, surgical site infection due to Mycobacterium mageritense and literature review, J Infect Dis Ther 2016;4:1-2

3. Gira AK, Reisenauer AH,Hammock L, Nadiminti U, Jonathan TM, Reeves A, Burnett C, Itchell M, Akrus Y, Toney S, Jensen BJ, Blumberg HM, Caughman SW, Frederick S, Furunculosis due to Mycobacterium mageritense associated with footbaths at a Nail Salon, J Clin Microbiol, 2004 ; 42(4):1813-1817.

4. Winthrop KL, Abrams M, Yakrus I, Schwartz JE, Gillies D, Vugia DJ, An outbreak of mycobacterial furunculosis associated with footbaths at a nail salon, N. Engl. J. Med, 2002; 346:1366-1371.

5. Croce O, Robert C, Raoult D, Drancourt M, Draft genome sequence of Mycobacterium mageritense DSM 44476 ${ }^{\mathrm{T}}$ Genome Announc, 2014; 2(2):00354-14.

6. Appelgren P, Filip, Leif D, Marie S, Bodil J, Bjorn P, Late-onset posttraumatic skin and soft-tissue infections caused by rapidgrowing Mycobacteria in Tsunami survivors, Clinical Infectious Diseases, 2008; 47(2):11-16. left side tubular ligation at site of stitch line (fig 5a). Advanced microbiological and molecular techniques, confirmed it as M. mageritense and anti-NTM treatment was given for seven months for complete recovery (fig 5b). To our knowledge, this is the first report of isolating and identifying M. mageritense at surgical site of a patient from India.

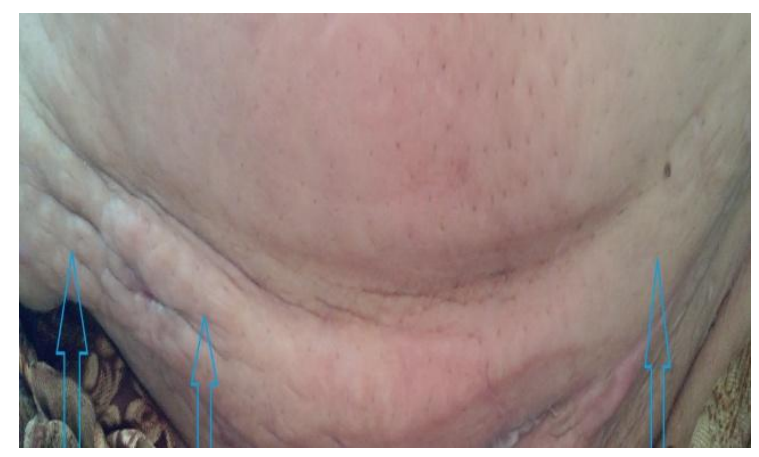

Figure 5b: After treatment

7. Wallace RJ, Brown-EBA, Hall L, Roberts G, Wilson RW, Mann LB, Crist CJ, Chiu SH, Dunlap R, Garcia MJ, Bagwell JT, Jost KC, Clinical and laboratory features of Mycobacterium mageritense, J Clin Microbiol, 2002; 40(8):2930-2935.

8. Ali S, Khan FA, Fisher M, Catheter-related bloodstream infection caused by Mycobacterium mageritense, J. Clin. Microbiol, 2007; 45:273. Doi,10.1128/JCM.01224-06.

9. Miki M, Shimizukawa M, Okayama H, Kazumi Y, Case of pulmonary Mycobacterium mageritense infection: the difficulty of differential diagnosis of granulomatous lung disease, Kekkaku, 2007; 82:189-194.

10. Morick D, Kik M, de BJ, Zanden AJM, Houwers DJ, Isolation of Mycobacterium mageritense from the lung of a Harbor Porpoise (Phocoena phocoena) with Severe Granulomatous Lesions, Journal of Wildlife Diseases, 2008; 44(4):999-1001.

11. Yonetani S, Kazumi Y, Araki K ,Hiroi M , Okuyama T, Ida Y, Makino $\mathrm{H}$,Takagi $\mathrm{Y}$, Ofuji M, Ohnishi H, Watanabe T, A Case of surgical site infection by Mycobacterium mageritense after gastrectomy, The Journal of Japanese Society for Clinical Microbiology, 2013; 23(2):112-116.

12. Maruyama T, Dougan SK, Truttmann MC, Bilate AM, Ingram JR, Ploegh HL, Increasing the efficiency of precise genome editing with CRISPR-Cas 9 by inhibition of nonhomologous end joining, Epub, 2015; 23.

13. Shah BS, Shah VV, Ray HSP, Shah PS, Shah ND, Katudia HK, Panchal YY, Khatri BK, Bhatiya RB, Toshniwal H, Patel BV, Shah SC, Rao MV, first isolation of mycobacterium farcinogenes in an Indian clinical specimen using rapid and recent technologies, EJBPS, 2018; 5(10):385-391.

14. Galkiewicz JP, Kellogg CA. Cross-kingdom amplification using bacteria-specific primers: complications for studies of coral microbial ecology. Appl Environ Microbiol. 2008; 74:78287831. 